\title{
Downscaling of the Climatic Change in the Mei-yu Rainband in East Asia by a Pseudo Climate Simulation Method
}

\author{
Hiroaki Kawase ${ }^{1}$, Takao Yoshikane ${ }^{1}$, Masayuki Hara ${ }^{1}$, Borjiginte Ailikun², \\ Fujio Kimura, ${ }^{1,3}$, and Tetsuzo Yasunari ${ }^{1,4}$ \\ ${ }^{1}$ Frontier Research Center for Global Change, JAMSTEC, Yokohama, Japan \\ ${ }^{2}$ Institute of Atmospheric Physics, Chinese Academy of Science, Beijing, P.R. China \\ ${ }^{3}$ Graduate School of Life and Environmental Sciences, University of Tsukuba, Tsukuba, Japan \\ ${ }^{4}$ Hydrospheric Atmospheric Research Center, Nagoya University, Nagoya, Japan
}

\begin{abstract}
This study focuses on the reproducibility of the climatic change in the early summer rainfall in East Asia (Mei-yu rainband) by the Pseudo Climate Simulation (PCS) using a regional climate model. In the PCS, the lateral boundary condition is given by the composite of six hourly reanalysis data and the difference between two decadal climate means. The daily variation on the lateral boundary of the PCS is similar to that of the control hindcast in one decade, but its climate is the same as the other decadal mean. The PCS accurately reproduces the change in the Mei-yu rainband over Southern China between the 1960s and the 1990s, suggesting that the climatic features of the Mei-yu rainband are controlled by the climatic change in a large-scale circulation.

The PCS reduces the uncertainty caused by the interannual variability in case of the downscaling of global warming projected by the General Circulation Models (GCMs) even if the number of sample years is the same as that in the conventional dynamical downscaling. The PCS can also exclude model biases in the present climatology reproduced by each GCM.
\end{abstract}

\section{Introduction}

To project the climatic change in a specific area, some methods have been proposed, such as the dynamical and statistical downscaling (Murphy 1999; Wood et al. 2004; Wang et al. 2004). The conventional method of dynamical downscaling (hereafter; referred to as the direct dynamical downscaling) consists of some numerical simulations using a regional climate model (RCM) assuming the initial and lateral boundary conditions given by the General Circulation Models (GCMs). A new concept of the dynamical downscaling has been introduced by Kimura and Kitoh (2007) and Sato et al. (2007). This method is almost the same as the direct dynamical downscaling but the lateral boundary condition of the $\mathrm{RCM}$ is a composite of the present reanalysis data and the difference of monthly mean values between the present climate and the future climate simulated by a GCM. Sato el al. (2007) conducted the future climate projection in Mongolia using the new downscaling method.

However, it has not been confirmed that this new downscaling method can simulate the past climatic change. The purpose of this study is to investigate the reproducibility of the past climatic change by the new downscaling method. We call it the Pseudo Climate Simulation (PCS) in this paper. We focused on the climatic change in the early summer rainfall over

Corresponding author: Hiroaki Kawase, Frontier Research Center for Global Change, Japan Agency for Marine-Earth Science and Technology, 3173-25, Showa-machi, Kanazawa-ku, Yokohama, Kanagawa, 236-0001, Japan. E-mail: kawase@ jamstec.go.jp. (C2008, the Meteorological Society of Japan.

\section{Southern China.}

\section{Mei-yu rainband in Southern China}

A large rainband, called the Mei-yu rainband in China, brings a large amount of rainfall over Southern China in summer (Kurashima and Hiranuma 1971), especially over the Yangtze River Basin (YRB) in early summer (June). Recently, the total summer rainfall has been increasing over the YRB (Endo et al. 2005). Gong and Ho (2002) speculated that the extension and intensification of the subtropical northwestern Pacific High (hereafter, Pacific High) since 1980 were closely related to the increase in precipitation using the six-hourly National Center for Environmental Prediction (NCEP)/ National Center for Atmospheric Research (NCAR) reanalysis data.

Figure 1a shows the ten-year mean precipitation observed in June during the 1990s. In situ observation

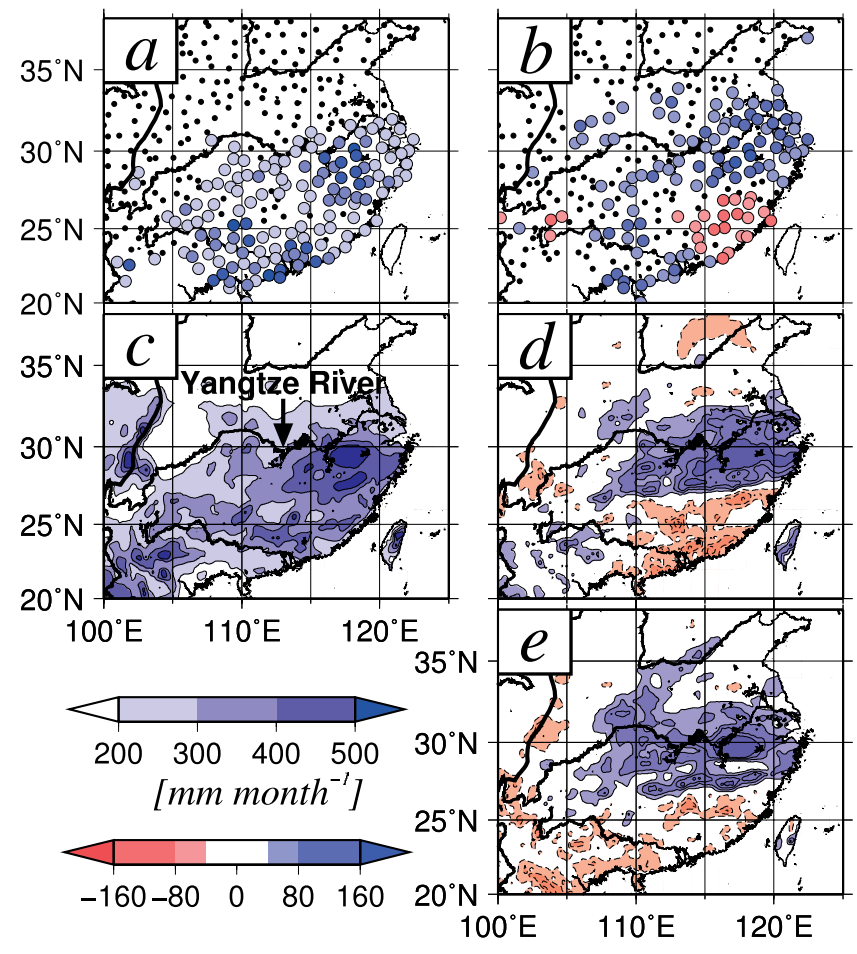

Fig. 1. Distributions of ten-year mean monthly precipitation (unit: $\mathrm{mm}$ month $^{-1}$ ) in June: (a) observations at the stations in the 1990s and (c) simulation by the HC-1990s. Differences in precipitation (b) between observations in the 1960s and the 1990s, (d) between the HC-1960s and the HC-1990s, and (e) between the PCS-1960s and the HC-1990s. In Figs. 1b, 1d, and $1 \mathrm{e}$ blue (red) shading indicates that precipitation increased (decreased) from the 1960 s to the 1990 s. 
Table 1. Specifications of simulations.

\begin{tabular}{cccc}
\hline Run Names & Initial and lateral boundary conditions & Period & Description \\
\hline HC-1960s & ERA40 & $1960 \mathrm{~s}$ & Hindcast \\
HC-1990s & ERA40 & $1990 \mathrm{~s}$ & Hindcast \\
PCS-1960s & pseudo ERA40 & pseudo 1960s & Pseudo Climate Simulation \\
\hline
\end{tabular}

a The change of large-scale atmospheric circulation between the 1960s and the 1990s is subtracted from every six-hour ERA40 in the 1990s.

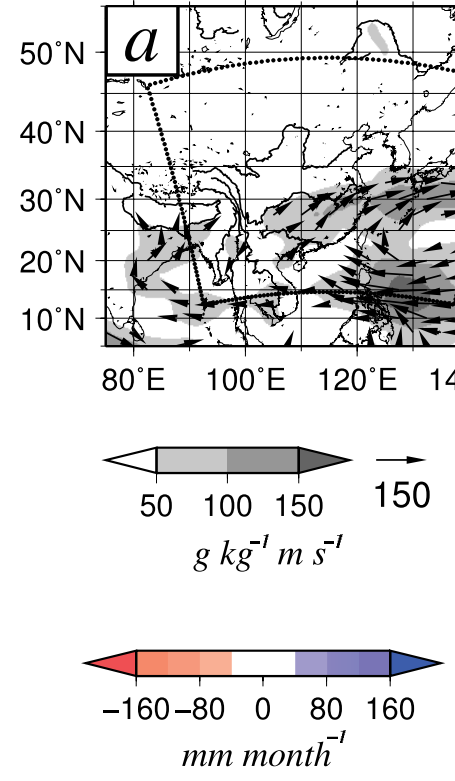

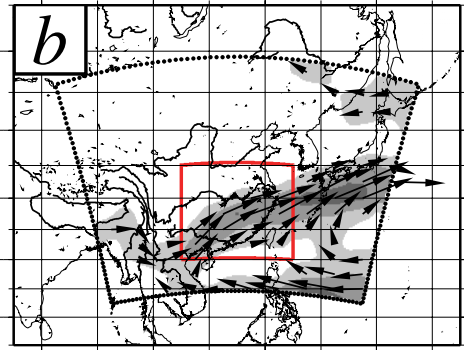
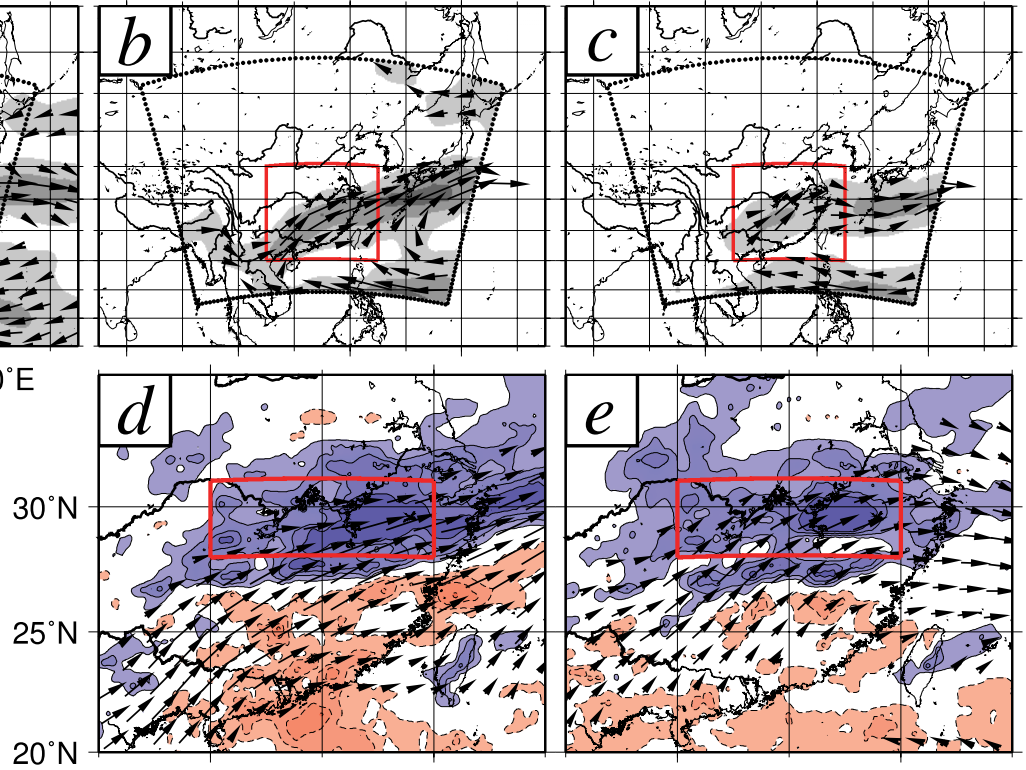

$110^{\circ} \mathrm{E}$

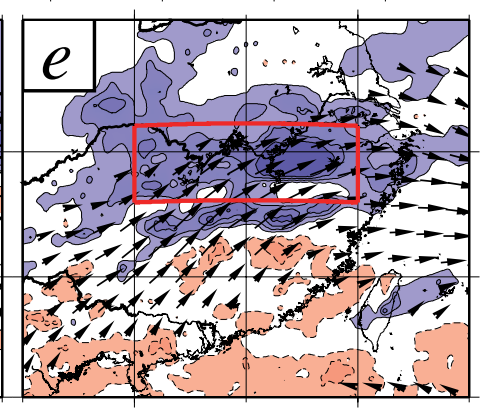

$110^{\circ} \mathrm{E}$

$120^{\circ} \mathrm{E}$

Fig. 2. Differences in the monthly mean moisture flux in June (unit: $\mathrm{g} \mathrm{kg}^{-1} \mathrm{~m} \mathrm{~s}^{-1}$ ) (a) between the 1960s and 1990s derived from the ERA40, (b) between the HC-1960s and HC-1990s, and (c) between the PCS-1960s and HC-1990s. The grid intervals in Figs. 2b and $2 \mathrm{c}$ are interpolated to 2.5 degrees, as are those of the ERA40. The region enclosed by black lines in Figs. 2a, $2 \mathrm{~b}$, and $2 \mathrm{c}$ represents the model domain. Figures $2 \mathrm{~d}$ and $2 \mathrm{e}$ show the differences in the mean monthly precipitation (shade; unit: $\mathrm{mm} \mathrm{month}^{-1}$ ) and moisture flux (vector; unit: $\mathrm{g} \mathrm{kg}^{-1} \mathrm{~m} \mathrm{~s}^{-1}$ ) in the regions enclosed by red lines in Fig. $2 \mathrm{~b}$ and in Fig. 2c.

data were provided by the China Meteorological Administration. Heavy precipitation exceeding $350 \mathrm{~mm} /$ month occurred south of the Yangtze River $(27.5 \mathrm{~N}$, $117.5 \mathrm{E})$ and around the southern coast of China. Figure $1 \mathrm{~b}$ shows the differences in the ten-year mean precipitation in June between the 1960s and the 1990s. Precipitation increased around the Yangtze River but decreased in the southern adjacent area.

The vertically integrated water vapor flux (hereafter, moisture flux) is estimated in June during the 1990s by the ECMWF 40-year Re-Analysis Data (ERA40). The distribution of the moisture flux shows the typical pattern in the Mei-yu season suggested by Kurashima and Hiranuma (1971) and Ninomiya (2000) (see Supplement 1a). Figure 2a shows the difference in the moisture flux derived from the ERA40 between the 1960 s and the 1990s. A clear anti-cyclonic circulation anomaly is found over the northwestern Pacific Ocean in the 1990s, which is consistent with a report by Gong and Ho (2002). The extension of the Pacific High appears not only in the NCEP/NCAR reanalysis data but also in the ERA40.

\section{Design of the Pseudo Climate Simulation}

The Advanced Research of Weather Research and Forecasting (WRF) model, version 2.2 (Skamarock et al. 2005), was used in this study. The domain of the nu- merical simulation was covered by $100 \times 130$ grids at 40-kilometer grid intervals (Fig. 2a). In order to simulate the precipitation in June, the simulation was executed each year from May 22 to July 1 . Both the Kain-Fritsch convective parameterization scheme (Kain and Fritsch 1993) and the WRF single-moment microphysics scheme (WSM6) were activated.

The procedure of the past PCS is as follows. Ten-year monthly mean fields of the ERA40, including the wind, temperature, geopotential height, and sea surface temperature, were estimated in June in the 1960s and the 1990s. The component of climatic change between the 1960 s and the 1990s was obtained as the difference between them. The components of climatic change were subtracted from each six-hour ERA40 in the 1990s, and the pseudo reanalysis data corresponding to the 1960s (hereafter, P-ERA40-1960s) were then obtained. The relative humidity of the P-ERA40-1960s was assumed to be equal to that of the ERA40 in the 1990s, which means that the amount of water vapor was expected to change only due to the change in temperature because the saturation vapor pressure increases with temperature (i.e., the Clausius-Clapeyron relation).

We conducted the PCS run in the 1960s (PCS-1960s) adapting the P-ERA40-1960s to the lateral boundary conditions of the WRF. This paper assumes that the interannual variability does not change in a different climate. The hindcast runs for the 1960s and the 1990s (HC-1960s and HC-1990s, respectively) were also con- 
ducted in comparison with the PCS run. The specifications of the simulations are summarized in Table 1.

\section{Simulated precipitation and moisture flux}

The distribution of precipitation was simulated well in the hindcast run, HC-1990s (Fig. 1c). The maximum precipitation in the domain was consistent with the observation (Fig. 1a), while precipitation was overestimated for all of Southern China. Figure 1d shows the difference between the HC-1960s and the HC-1990s, which indicates an increase in precipitation around the YRB and a decrease in precipitation to the south of the YRB from the 1960s to the 1990s. These results agree with the observed precipitation change shown in Fig. $1 \mathrm{~b}$.

Figure 1e shows the difference between the precipitation in the PCS-1960s and that in the HC-1990s. The difference of the precipitation is similar to the observed one, as shown in Fig. 1b, and also similar to the difference between the two hindcast runs, as shown in Fig. $1 d$, especially for the increase in precipitation around the YRB and the decrease to the south of the YRB. These results indicate that the precipitation change between the 1960s and the 1990s can be simulated well by the PCS run.

The decadal mean large-scale moisture fluxes in June are simulated well by the HC-1960s and HC-1990s although they are slightly overestimated (see Supplement 1). Figure $2 \mathrm{~b}$ shows the difference of the moisture flux between the HC-1960s and the HC-1990s. The anticyclonic circulation anomaly can be reproduced over the northwestern Pacific Ocean as well as the ERA40 (Fig. 2a). This indicates that the RCM does not strongly modify the large-scale system.

Figure $2 \mathrm{c}$ is the same as Fig. $2 \mathrm{~b}$ but shows the difference between the PCS-1960s and the HC-1990s. The anti-cyclonic circulation anomaly is also simulated over the northwestern Pacific Ocean, which is quite similar to Fig. $2 \mathrm{a}$ and Fig. 2b. It is noteworthy that the daily variation of the lateral boundary in the PCS-1960s differs from that in the HC-1960s but the decadal climates of their lateral boundaries are the same. These results suggest that the decadal change in the moisture flux in the model domain is strongly affected by the climatic forcing from the lateral boundary of the RCM.

Ninomiya (2000) suggested that the differential advection of equivalent potential temperature, which consists of a poleward moisture flux and cold air advection by the upper-level subtropical jet, is the primary factor in the formation of the active Mei-yu rainband. Figures $2 \mathrm{~d}$ and $2 \mathrm{e}$ show the differences in the moisture flux and precipitation between the HC-1960s and the HC-1990s and between the PCS-1960s and the HC-1990s, respectively. The northeastward component of the moisture flow increases in the YRB in both cases. Precipitation increases in the northern part of the domain and decreases in the southern part of the domain. The northward shift of the upper subtropical jet at $200 \mathrm{hPa}$ is also simulated over Southern China between the PCS-1960 and the HC-1990s (figure not shown). The simulations suggest that the change in the moisture flux modulates the differential advection in association with the upper subtropical jet and results in the change in precipitation over Southern China.

\section{Discussion}

The climatic change was sufficiently simulated by the PCS on the early summer rainfall in China between the 1960 s and the 1990s. To confirm the reliability, another PCS run (PCS-1980s) was conducted for the period of the 1980s when precipitation was less than that in the 1990s in most of Southern China. The results
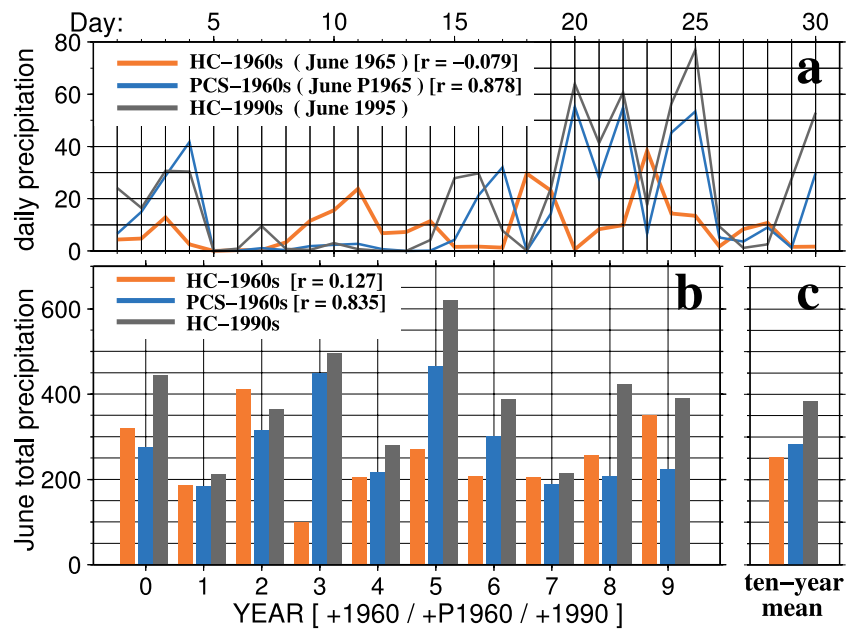

Fig. 3. Time series of the regional mean precipitation (unit: mm) over the enclosed area in Figs. 2d and 2e.

(a) Variations of the daily precipitation in June 1965 simulated by the HC-1960s (orange), pseudo 1965 simulated by the PCS1960s (light blue), and 1995 simulated by the HC-1990s (gray). (b) Interannual variation of total precipitation in June. (c) Tenyear mean precipitation in June.

showed that the difference between the PCS-1980s and the HC-1990 agreed well with the observed difference between the 1980s and the 1990s as well as with that between the HC-1980s and HC-1990s although the PCS1980s underestimated the magnitude of the differences (see Supplement 2).

Figure 3 is a time series of regional mean precipitation over the enclosed area in Figs. $2 \mathrm{~d}$ and 2e. The upper panel (a) shows the variation of the daily precipitation in June 1965 (HC-1960s), pseudo 1965 (PCS-1960s), and 1995 (HC-1990s). Lower panels (b) and (c) show the interannual variation of the monthly total precipitation and ten-year mean precipitation in June, respectively. The daily variation of precipitation in the HC-1990s is quite similar to that in the PCS-1960s (Fig. 3a), whose correlation coefficient is 0.878 . The large positive correlation is considered to be induced by the common shortterm components in the boundary conditions using in the HC-1990s and the PCS-1960s. On the other hand, the HC-1960s shows different behavior from the other simulations. The behavior in the HC-1960s is almost independent from that in the HC-1990s, as expected. In the same way, the interannual variations of precipitation in the PCS-1960s are similar to those in the HC-1990s, whose correlation coefficient is 0.835 (Fig. $3 \mathrm{~b}$ ), although the amount of precipitation in the PCS-1960s is always less than that in the HC-1990s. The large correlation coefficient results from the strong relationship between the HC-1990s and the PCS-1960 (see Section 3). In contrast, the correlation coefficient between the two hindcasts, the HC-1960s and the HC-1990s, is quite low.

On the other hand, the amount of ten-year mean precipitation in the PCS-1960s is $261.6 \mathrm{~mm}$, which is comparable to that estimated in the HC-1960s $(234.3 \mathrm{~mm})$ but much less than that in the HC-1990s (353.8 mm) (Fig. 3c). As reported in Section 4, the horizontal distribution of the difference in precipitation between the $\mathrm{HC}-1960 \mathrm{~s}$ and the HC-1990s is quite similar to that between the PCS-1960s and the HC-1990s (Figs. 1d and $1 \mathrm{e}$ ), as is the difference in the moisture flux (Figs. $2 \mathrm{~b}$ and 2c). These facts indicate that the climatic change estimated by the PCS-1960s is similar to that estimated by the HC-1960s. We concluded that the time evolution of the PCS-1960s was different from that of the HC-1960s but the characteristics of the climate of the PCS-1960s were similar to those of the HC-1960s. 
These characteristics of the PCS run bring two major advantages for the case of the downscaling of global warming projected by GCMs. One is the reduction of the uncertainty caused by the interannual variability, and the other is the reduction of the model bias contained in the projection by GCMs, although the reduction is limited in the component of the climate mean.

If we assume no correlation between the two groups of annual mean values sampled from two datasets, the estimated standard deviation of the difference between the two mean values depends only on the number of sample years and the standard deviations of the original datasets. In the case of the direct dynamical downscaling, e.g., the hindcast runs in this paper, the two data groups, the HC-1960s and the HC-1990s, are expected to have a low correlation; thus, a larger number of sample years are required for the smaller uncertainty caused by the interannual variability. In case of the PCS, e.g., the PCS-1960s and the HC-1990s, however, the estimated standard deviation of the difference becomes smaller because of the strong correlation between the two datasets even if the number of sample years is the same as that in the direct dynamical downscaling.

The second advantage was also reported by Sato et al. (2007). The direct dynamical downscaling is contaminated by the model biases of the GCM. On the other hand, the lateral boundary condition of the PCS consists of the present reanalysis data and the components of climatic change estimated by the GCMs. The PCS is contaminated only by the uncertainty of the climatic change estimated by the GCM since the present climate is replaced by the reanalysis data.

The PCS has disadvantages, however. In the PSC runs, the frequency of the large-scale disturbance would be difficult to change, although their intensity may change. The PCS assumes that the interannual variability does not change in a different climate. The PCS, however, would contribute to the reduction of the uncertainty of the downscaling method as one of the different tools from the direct dynamical downscaling.

When the PCS is applied to the future projection, the lateral boundary condition is given by the composite of the six hourly present reanalysis data and the difference between the present climate and the future climate projected by GCMs. The difference would be better to be estimated by the more than ten-year means of the present and future climates or by the model ensemble means in order to reduce the natural variabilities, such as interannual and decadal variabilities. Even in these cases, short-term integrations, e.g., the two ten-year integrations in this paper, by the PCS method would reduce the uncertainty caused by the natural variability in comparison with the integrations by the direct dynamical downscaling methods.

\section{Conclusion}

The reproducibility of the climatic change was evaluated by the PCS method. The PCS method can reproduce some decadal changes in the rainfall observed over Southern China. This indicates that the change in the large-scale circulation contributes to the climatic change in the rainfall associated with the Mei-yu rainband through the climatic forcing from the lateral boundary of the RCM.

The PCS method has some advantages to evaluate the future regional climatic change. This method would reduce the uncertainty caused by the natural variability in the simulated climatic change in comparison with the direct dynamical downscaling method because the interannual variation of the PCS correlates well with that of the control hindcast. The PCS can also exclude model biases in the present climatology reproduced by each GCM. In short, the PCS would contribute to the reduction of the uncertainty of the downscaling method as one of the different tools from the direct dynamical downscaling.

\section{Acknowledgments}

This work was supported by the Global Environment Research Fund (B-061) of the Ministry of the Environment, Japan. This work was partly supported by the Ministry of Education, Culture, Sports, Science and Technology (MEXT). It was also supported by MEXT through a Grant-in-Aid for Scientific Research (Category B, 17310003). One of the authors (Kimura) was supported by the Global Environment Research Fund (S-5-3) of the Ministry of the Environment, Japan. This research was jointly supported by the China National Key Planning Development for Basic Research (Grant No.2006CB400504). The authors thank Dr. Endo of the Institute of Observational Research for Global Change for his meaningful comments about the precipitation dataset. We thank two anonymous reviewers for their helpful comments.

\section{Comments and supplements}

1. Monthly mean moisture fluxes in June.

2. Distributions of the ten-year mean monthly precipitation in June during the 1980s.

\section{References}

Endo, N., B. Ailikun, and T. Yasunari, 2005: Changes in precipitation amounts and the number of rainy days and heavy rainfall events during summer in China. J. Meteor. Soc. Japan, 83, 621-631.

Gong, D., and C. Ho, 2002, Shift in the summer rainfall over the Yangtze River valley in the late 1970s, Geophys. Res. Lett., 29(10), 1436, doi:10.1029/2001GL014523.

Kain, J. S., and J. M. Fritsch, 1993: Convective parameterization for mesoscale models: The Kain-Fritsch scheme. The representation of cumulus convection in numerical models, Amer. Meteor. Soc., K. A. Emanuel and D. J. Raymond, Eds., 246 pp.

Kimura, F., and A. Kitoh, 2007: Downscaling by Pseudo Global Warming Method, The Final Report of ICCAP, 43-46.

Kurashima, A., and Y. Hiranuma, 1971: Synoptic and climatological study on the upper moist tongue extending from Southeast Asia to East Asia. Water Balance of Monsoon Asia, University of Tokyo Press, M. M. Yoshino, Ed., 153 -169 .

Murphy, J., 1999: An evaluation of statistical and dynamical techniques for downscaling local climate. J. Climate, 12, 2256-2284.

Ninomiya, K., 2000: Large- and meso- $\alpha$-scale characteristics of Meiyu/Baiu Front associated with intense rainfalls in 1.10 July 1991. J. Meteor. Soc. Japan, 78, 141-157.

Sato, T., F. Kimura, and A. Kitoh, 2007: Projection of global warming onto regional precipitation over Mongolia using a regional climate model. J. Hydrol., 333, doi:10.1016/ j.jhydrol.2006.07.023.

Skamarock, W. C., J. Dudhia, D. O. Gill, D. M. Barker, W. Wang, and J. G. Powers, 2005: A description of the Advanced Research WRF version 2, NCAR Tech. Rep., TN-468, 100 pp.

Wang, Y. Q., L. R. Leung, J. L. McGregor, D.-K. Lee, W.-C. Wang, Y. H. Ding, and F. Kimura, 2004: Regional climate modeling: progress, challenges, and prospects. J. Meteor. Soc. Japan, 82, 1599-1628.

Wood, A. W., L. R. Leung, V. Sridhar, and D. P. Lettenmaier, 2004: Hydrologic implications of dynamical and statistical approaches to down-scaling climate model outputs. Clim. Change, 62, 233-256.

Manuscript received 4 June 2008, accepted 28 July 2008

SOLA: http://www.jstage.jst.go.jp/browse/sola/ 